\title{
DETERMINAÇÃO DOS TEORES DE ÁCIDO ACETILSALICÍLICO EM AMOSTRAS DE PLASMA POR ESPECTROFOTOMETRIA
}

\author{
Dandara Antunes COIMBRA ${ }^{1}$ \\ Mirelle Oliveira SÓTER ${ }^{2}$ \\ Daniela Fernanda FREITAS ${ }^{2,3}$
}

1. Discente, Centro Universitário de Lavras, Curso de Graduação em Farmácia Generalista;

2. Docente, Centro Universitário de Lavras, Curso de Graduação em Farmácia Generalista; mirellesoter@yahoo.com.br;

3. Docente, Faculdade de Ciências e Tecnologias de Campos gerais, Curso de Graduação em Farmácia

Generalista.danielaffreitas@bol.com.br

\section{Recebido em: 25/10/2013 - Aprovado em: 1712/2013 - Disponibilizado em: 15/01/2014}

RESUMO: O monitoramento terapêutico constitui atividade sistemática de análise de fármacos e seus metabólitos ativos em material biológico, com o objetivo de se obter a máxima eficácia terapêutica com mínimo ou ausência de efeitos tóxicos. É recomendada para pacientes que usam Ácido Acetilsalicílico (AAS) de forma crônica, devido à alta variabilidade interindividual das concentrações plasmáticas e a baixa adesão de alguns pacientes. Assim, o objetivo deste trabalho foi o de determinar os teores de AAS em amostras de plasma, de pacientes em uso de ácido acetilsalicílico (100mg), atendidos no Programa de Saúde da Família (PSF 14) de Lavras - MG visando monitorização terapêutica. Foram selecionados 10 pacientes, maiores de 18 anos e de ambos os sexos, sob uso de AAS $100 \mathrm{mg}$ em diferentes regimes de dosagem. Essas amostras foram coletadas imediatamente antes da ingestão da próxima dose (concentração vale) e foram analisadas pelo método espectrofotométrico de Trinder, ( $\lambda$ 540nm). Para cálculo de teor de AAS em plasma utilizou-se a equação $(y=0,0197 x+0,0157)$, com um coeficiente de determinação $\left(\mathrm{R}^{2}\right)$ de 0,9996 . Foram encontrados valores entre 0,0 e $3,67 \mathrm{mg} \%$. Metade dos pacientes analisados, provavelmente, não estava aderindo ao tratamento ou ainda estavam em dose subterapêutica; o que pode ser justificado pela alta frequência com que são relatados os efeitos adversos. O método de Trinder é fácil, rápido e aplicável para prática clínica. Mas ainda é necessária a determinação de valores de referência específicos para a dosagem de AAS no plasma, quando este é administrado em baixas doses (efeito anticoagulante).

PALAVRAS CHAVE. Monitorização terapêutica. Coronáriopatias. Espectrofotômetro. Ácido Acetilsalicílico.

\begin{abstract}
The therapeutic monitoring activity is systematic analysis of drugs and their active metabolites in biological material, with the goal of achieving maximal therapeutic efficacy with minimal or no toxic effects. It is recommended for patients using Aspirin (ASA) chronically, due to high between individual variability in plasma concentrations and poor adherence in some patients. The objective of this work was to determine the levels of ASA in plasma samples of patients using aspirin (100mg), served in the Family Health Program (PSF 14) Lavras - MG, targeting therapeutic monitoring. We selected 10 patients, aged 18 years and of both sexes, under ASA 100 mg in different dosing regimens. These samples were collected immediately before the intake of the next dose (concentration valley) and were analyzed by the spectrophotometric method of Trinder ( $\lambda 540 \mathrm{~nm}$ ). To calculate the amount of ASA in plasma we used the equation $(\mathrm{y}=0.0197 \mathrm{x}+0.0157)$ with a coefficient of determination $\left(\mathrm{R}^{2}\right)$ of 0.9996 . Values were between 0.0 and $3.67 \mathrm{mg} \%$. Half of the patients analyzed, probably was not adhering to treatment or were still subtherapeutic dose, which can be explained by the high frequency with which adverse effects are reported. The method of Trinder's easy, fast and applicable to clinical practice. But it is still necessary to determine reference values for specific dosing of ASA in plasma when administered at low doses (anticoagulant effect).
\end{abstract}

KEYWORDS. Therapeutic drug monitoring. Coronary Artery Disease. Spectrophotometer. Aspirin.

\section{INTRODUÇÃO}

O monitoramento terapêutico constitui

atividade sistemática de análise de fármacos

e/ou seus metabólitos ativos em material biológico, geralmente o plasma, a fim de se obter a máxima eficácia terapêutica com mínimo ou ausência de efeitos tóxicos. Os fármacos que requerem o emprego de monitorização terapêutica normalmente 
apresentam estreita faixa terapêutica, pouca correlação entre dose administrada e resposta terapêutica, grandes variações farmacocinéticas, sintomas tóxicos não específicos e resposta terapêutica tardia (STEIMER et al, 2001; SANTOS, 2008).

A monitorização terapêutica requer usualmente especificidade para distinguir o fármaco de seus metabólitos similares quimicamente ou de outros fármacos coadministrados. Assim, os seguintes quesitos são necessários para se ter um eficiente preparo da amostra: a) perda mínima da amostra e boa recuperação do analito; b) componentes existentes na amostra e sem interesse devem ser removidos eficientemente; c) não devem ocorrer problemas no detector; d) o procedimento deve ser feito de forma conveniente e rápido; e) o custo da análise deve ser baixo (KATAOKA, LORD, 2002).

O emprego sistemático do monitoramento terapêutico, que se trata do controle das concentrações plasmáticas de um fármaco com a finalidade de ajuste de dose e individualização da terapia farmacológica, pode minimizar as variações que ocorrem nas concentrações sanguíneas dos fármacos $\mathrm{e}$ reduzir seus efeitos adversos; sendo também útil para avaliar a adesão do paciente ao tratamento (SANTOS, 2008).

Santos (2008) ainda descreve que esta atividade é indicada para o uso crônico de fármacos. Dentre os grupos de fármacos para os quais esta atividade pode ser realizada destaca-se os salicilatos, entre eles, o ácido acetilsalicílico, a aspirina e outros antiinflamatórios não esteróides (AINEs). Segundo Rang et al, (2007), estes fármacos são utilizados como analgésicos, antitérmicos e antiinflamatórios, também são empregados no tratamento de moléstias reumáticas e na prevenção de doenças cardiovasculares atuando como antiagregante plaquetário.

Contudo, a monitorização terapêutica é um procedimento recomendado para pacientes que usam AAS, devido seu uso crônico na prevenção de coronariopatias, bem como, à alta variabilidade interindividual das concentrações plasmáticas e a baixa adesão de alguns pacientes, devido aos efeitos adversos comuns ao uso de Aspirina.

Uma grande variedade de métodos analíticos tem sido desenvolvida para a determinação de concentrações de salicilatos nos fluidos e tecidos biológicos. Assim, análises seguras e rápidas de salicilato no sangue são de suma importância em medicina, bem como o controle de qualidade do ácido acetilsalicílico em sua produção (ROVER JÚNIOR, 1999).

Em análises clínicas, as técnicas colorimétricas têm sido muito empregadas pela relativa facilidade na determinação de salicilato. O teste de Trinder (TRINDER, 1954; KANG et al, 1983) é o método colorimétrico mais usado entre todas as metodologias espectrofotométricas. Cromatografia gás-liquído (CGL) e cromatografia líquida de alta eficiência 
(CLAE) têm sido empregadas para separação e quantificação simultânea de ácido salicílico, ácido o-acetil-salicílico e outros compostos presentes em diversas formulações farmacêuticas.

O presente trabalho teve por objetivo determinar os teores de ácido acetilsalicílico em amostras de plasma, de pacientes em uso de ácido acetilsalicílico (100mg), atendidos no Programa de Saúde da Família (PSF 14) de Lavras - MG, visando monitorização terapêutica.

\section{MATERIAL E MÉTODOS}

Este trabalho foi conduzido de março de 2011 a março de 2012 no Laboratório de Química do Centro Universitário de Lavras. A pesquisa foi realizada sob os preceitos éticos determinados pela resolução 196/96 do Conselho Nacional de Saúde e sob aprovação do Comitê de ética do UNILAVRAS (0113.0.189.000-10 - 22/02/2011).

Para realização da monitorização terapêutica, foram selecionados 10 pacientes sob uso de AAS 100mg, maiores de 18 anos e de ambos os sexos, cadastrados no Programa de Saúde da Família 14 (PSF-14), em LavrasMG.

As amostras de plasma, dos pacientes sob uso de AAS, foram obtidas dos voluntários, em diferentes regimes de dosagem e antes da ingestão da próxima dose (concentração vale). Coletou-se cerca de 20 $\mathrm{mL}$ de sangue venoso e, em seguida, realizou- se a centrifugação da amostra e separação do plasma que foi conservado a $-20^{\circ} \mathrm{C}$ até sua análise.

O método utilizado foi o de Trinder, método colorimétrico no qual a concentração de ácido salicílico (e não ácido acetilsalicílico) é diretamente proporcional à intensidade de coloração violeta do complexo formado entre salicilato e íons férrico.

Para a curva analítica foram utilizadas as seguintes concentrações de ácido salicílico: 0,$05 ; 0,1 ; 0,5 ; 1,0 ; 5,0 ; 10 ; 20 ; 30$ e 40 mg\%, o desvio padrão entre as replicatas variaram entre $0,0020-0,0062 \%$.

\section{RESULTADOS E DISCUSSÃO}

\section{A FIGURA 1 ilustra a Curva Analítica} utilizada para calcular os resultados obtidos na pesquisa. Os dados apresentados evidenciam linearidade satisfatória no intervalo selecionado, com coeficiente de determinação de 0,9996 .

Figura 1 - Curva Analítica construída com padrão de ácido salicílico.

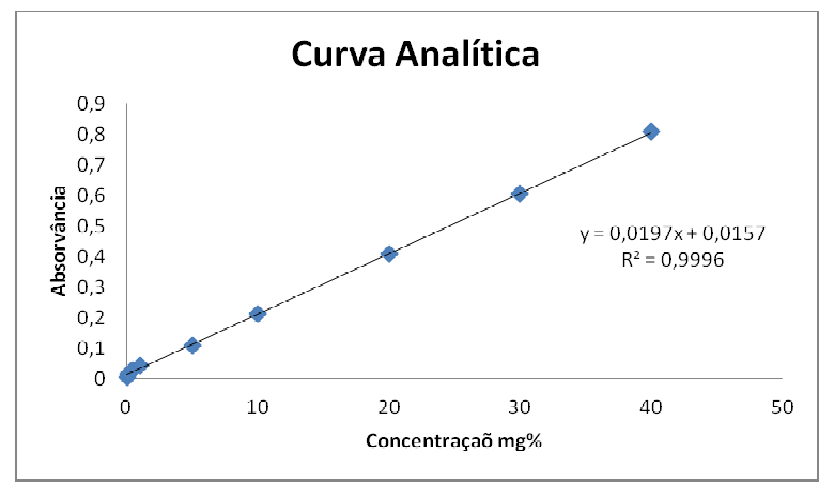

Os resultados relativos à determinação dos teores de ácido salicílico estão 
expressos na tabela 1. O Paciente 9 apresentou maior concentração plasmática de ácido salicílico, quando comparado aos pacientes 1 e 8 , mesmo após administração do medicamento uma única vez ao dia. Os pacientes 1 e 8 faziam uso de 2 comprimidos de AAS/dia enquanto que o paciente 9 fazia uso de apenas um comprimido de AAS/dia. Observa-se que não houve correlação entre a quantidade de comprimidos administrados e a concentração encontrada no plasma, evidenciando que a variabilidade interindividual é um fator importante na avaliação da eficácia do tratamento.

Tabela 1: Concentração de Ácido salicílico em amostras de plasma de pacientes em uso de AAS 100 mg.

\begin{tabular}{|c|c|c|c|}
\hline Paciente & Regime de dosagem & $\begin{array}{c}\text { Média das } \\
\text { bbsorbâncias }\end{array}$ & $\begin{array}{c}\text { Concentração } \\
(\mathbf{m g} / \mathbf{1 0 0 m L})\end{array}$ \\
\hline 1 & 2 comprimidos/dia & 0,0214 & 0,29 \\
\hline 2 & 1 comprimido/dia & 0,0078 & 0 \\
\hline 3 & 2 comprimidos/dia & 0,0152 & 0 \\
\hline 4 & 1 comprimido/dia & 0,0126 & 0 \\
\hline 5 & 4 comprimidos/dia & 0,0112 & 0 \\
\hline 6 & 1 comprimido/dia & 0,0182 & 0,127 \\
\hline 7 & 1 comprimido/dia & 0,0086 & 0 \\
\hline 8 & 2 comprimido/dia & 0,031 & 0,77 \\
\hline 9 & 1 comprimido/dia & 0,088 & 3,67 \\
\hline 10 & 1 comprimido/dia & 0,0207 & 0,25 \\
\hline
\end{tabular}

Em metade dos pacientes não foi encontrado o metabólito ácido salicílico, o que sugere a administração incorreta ou, até mesmo, a não administração das doses prescritas. Manifestações gastrointestinais tais como: intolerância gástrica, náuseas, vômitos, flatulência e dor abdominal são comuns ao uso de AAS e estas têm contribuído para o uso incorreto deste medicamento, aumentando as chances de eventos tromboembólicos (RANG et al, 2007).

Porém, para confirmar a baixa adesão ao tratamento, seria necessária a pesquisa de outros metabólitos do ácido acetilsalicílico, no plasma ou urina; e o uso de técnicas mais sensíveis para dosagem do AAS administrado em baixas doses.

\section{CONCLUSÃO}

De acordo com os dados apresentados neste trabalho observa-se que cinco pacientes estavam em doses terapêuticas e alguns pacientes não estavam aderindo corretamente ao tratamento devido a determinação de dose subterapêutica do AAS; o que pode ser justificado pela alta frequência com os relatos dos efeitos adversos. Os resultados foram encaminhados ao médico do PSF-14 de Lavras-MG para posterior análise individual de cada paciente.

O método de Trinder é fácil, rápido e aplicável para prática clínica. Mas ainda é necessária a determinação de valores de referência específicos para a dosagem de AAS no plasma por espectrofotômetro.

\section{REFERÊNCIAS BIBLIOGRÁFICAS}

CAMARGO, E.G. et al. Aspirina em Baixa Dosagem em Pacientes Com Diabete Melito: Riscos e Benefícios em Relação às Complicações Macro e Microvasculares. Arquivo Brasileiro Endocrinologia e 
Metabolismo, 2007; 51/3.

CATELLA-LAWSON, F., et al.

Cyclooxygenase inhibitors and the antiplatelet effects of aspirin. N Engl J Med, 2004; 345: 1809-1817.

GOTO, Y. et al. Chromatogr., B: Biomed. Sci. Appl. 1998, 706, 329.

HARDMAN, J. G.; LIMBIRD, L. E.

Goodman \& Gilman: As bases farmacológicas da terapêutica. $10^{\mathrm{a}}$ ed. Rio de Janeiro: McGraw-Hill Interamericana, 2003. 1647p.

LAI K.C. et al. Lansoprasole for the prevention of recurrences of ulcer complications from long-term low-dose aspirin use.N Engl J Med., jun 2002; 346 (26): 2033-8

MALDONADO, V. B. Efeitos Microscópicos do Ácido Acetilsalicílico (Aspirina) e do Acetaminofeno (Tylenol) na Movimentação Dentária e nas Reabsorções Rediculares Associadas. 2009. 82p. Dissertação (Mestrado em Odontopediatria) - Faculdade de Odontologia de Ribeirão Preto/USP, Ribeirão Preto, 2009.

PATRONO, C. et al. Low-dose aspirin for the prevention of atherothrombosis. N Engl

JMed,2005;353:2373-83

RANG, H. P. et al. Rang \& Dale:

Farmacologia. 6. ed. Rio de Janeiro: Elsevier, 2007. 828p.

ROVER JR., L. et al. Talanta. 2000, 51, 547.

SANTOS, S. R. C. J. Monitorização Terapêutica. In: MOREAU, R. L. de M.; SIQUEIRA, M. E. P. B.; Toxicologia Analítica. Rio de Janeiro: Guanabara Koogan, 2008. Cap. 9, p. 91-99.

SILVA. L. L. da. Estudo comparativo entre agregação plaquetária por turbidimetria e impedância elétrica em relação a pacientes sob terapia antiplaquetária a base de ácido acetilsalicílico. 2010. 51 p. Dissertação
(Mestrado em Distúrbios do Crescimento Celular, Hemodinâmicos e da Hemostasia) Faculdade de Medicina da Universidade de São Paulo, São Paulo, 2010.

STEIMER, W., MULLER, B., LEUCHT S., KISSLING, W. Pharmacogenetics: a new tool in the management of antidepressive drug therapy. Clin. Chem., v. 308, p. 33-41, 2001.

TRINDER, P.; Biochem. J. 1954, 57, 301.

PIROLA, R.; BAREGGI, S. R.; DEBENEDITTIS, G.; J. Chromatogr., B: Biomed. Sci. Appl. 1998, 705, 309. 